\title{
The Cost of Treating Osteoporosis in a Managed Health Care Organization
}

SHETAL S. DESAI, BS, PharmD; BABETTE S. DUNCAN, PharmD, MBA, BCPS; and ALICE S. SLOAN, RPh

\begin{abstract}
OBJECTIVE: To measure the differences in direct health care costs and resource utilization among female enrollees in a health maintenance organization who were aged 45 through 65 years and had either osteoporosis or an osteoporosis-related fracture.

METHODS: One year of medical and pharmacy claims (October 1, 1998, to September $30,1999)$ from a mixed-model health plan located in the Midwest were evaluated. Diagnoses were determined from medical claims with ICD-9 codes specific to either osteoporosis or osteoporosis-related fracture. Aggregate costs specific to osteoporosis were compared to all costs incurred by the members regardless of the disease states.

RESULTS: We identified 600 women who had consumed a total of $\$ 4.6$ million in health care resources and $\$ 411,684$ in direct costs specifically related to osteoporosis. The highest total average disease-specific costs were found for women with a fracture ( $\$ 939$ per patient per year [PPPY]) compared to those with osteoporosis only (\$645 PPPY). Outpatient costs accounted for the highest percentage of mean total annual costs of care, representing up to $38 \%$ of the total health care resources consumed. Average medical costs for women with a fracture were highest for the 60 to 64 years age category, the oldest age category in the study population (\$17,403 PPPY, $P=.0379$ ). Estrogen was the most utilized drug for treatment of osteoporosis, accounting for $41 \%$ of the total osteoporosis-specific prescription utilization.
\end{abstract}

CONCLUSION: The costs of care for members with osteoporosis-related fractures were, on average, higher than for women with osteoporosis only. The component costs included outpatient services, inpatient services, and prescription costs. Women not receiving drug therapy for management of osteoporosis incurred slightly higher total health care costs than women who did not receive drug therapy for osteoporosis.

KEYWORDS: Women's health, Osteoporosis, Pharmacy claims, Health care outcomes, Cost

J Managed Care Pharm. 2003(9)2:142-49
Authors

SHETAL S. DESAI, BS, PharmD, is Clinical Pharmacy Manager, Wellpoint Pharmacy Management, West Hills, California. (At the time this article was written, Desai was a Managed Care Pharmacy Resident, AdvancePCS).; BABETTE S. DUNCAN, PharmD, MBA, BCPS, is Vice President, Centers for Health Improvement, and ALICE S. SLOAN, RPh, is Vice President of Account Management, AdvancePCS, Hunt Valley, Maryland.

AUTHOR CORRESPONDENCE AND REPRINT REQUESTS: Shetal S. Desai, BS, PharmD, Clinical Pharmacy Manager, Wellpoint Pharmacy Management, 8407 Fallbrook Ave., AF-7, West Hills, CA 91304. Tel: (818) 315-5085; Fax: (818) 313-5110,E-mail: shetal.desai@wellpoint.com

Copyright $\odot$ 2003, Academy of Managed Care Pharmacy. All rights reserved.

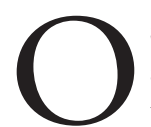
steoporosis is a growing public health concern associated with aging and is a major cause of morbidity and mortality in postmenopausal women. Characterized by low bone mass and microarchitectural deterioration of bone tissue, osteoporosis leads to bone fragility and an increased risk of developing a debilitating fracture. According to the National Osteoporosis Foundation, 13\% to $18 \%$ of postmenopausal white women in the United States have osteoporosis, and an additional $30 \%$ to $50 \%$ have low bone density at the hip. ${ }^{1}$ Postmenopausal women are at greatest risk of developing osteoporosis because of the accelerated loss in bone mass associated with the first several years of menopause. An estimated 1 of every 2 white women will experience an osteoporotic fracture at some point in her lifetime. ${ }^{1}$ The annual health care costs and resource utilization associated with osteoporosis are staggering, estimated to exceed $\$ 13$ billion. ${ }^{2}$

Currently, osteoporosis accounts for nearly 1.5 million fractures annually, with nearly 300,000 attributed to fractures of the hip. ${ }^{1}$ However, as the average life expectancy continues to increase, the frequency of developing fractures and the costs associated with this disease are expected to more than double by 2026 .

The consequences of osteoporosis, including fractures of the hip, wrist, and spine, can have devastating consequences on quality of life. One half of all hip-fracture patients will be unable to live independently, and hip fractures have been associated with a 12\% to $36 \%$ mortality rate within the first year of incident. ${ }^{1}$

In addition to the personal burden and impact on quality of life, the costs associated with fracture treatment and rehabilitation are enormous. Many countries, such as Australia and Switzerland, have estimated directs costs of osteoporosis for women; however, few cost-of-illness studies have been published in the United States and, thus, a need for such literature exists. ${ }^{3,4}$ At present, the most thorough estimate of the cost of osteoporosis was published by Ray and colleagues in $1997 .{ }^{2}$ This study, based on national health care survey data from 1995, estimated national health care expenditures attributable to osteoporosis fractures for persons aged 45 years and older to be $\$ 13.8$ billion. Approximately $75 \%$ of these costs ( $\$ 10.3$ billion) were for the treatment of white women and included direct medical costs associated with inpatient services, nursing home care, and outpatient services. However, studies estimating the average cost of treatment per patient per year (PPPY) or the proportion of women receiving antiosteoporotic agents for secondary fracture prevention in a managed health care setting are limited. Quantification of these costs may prove useful in the determination of cost drivers for this disease and in the allo- 
cation of limited resources and promotion of preventive health care services within a managed health care population.

The goal of this study was to determine the direct costs of treating osteoporosis and osteoporosis-related fractures and to identify the principal cost drivers and the prevalence of drug therapy utilization among women with either diagnosis. Using medical and pharmacy claims data, we expected to (a) estimate the direct health care expenditures associated with treating osteoporosis and osteoporosis-related fractures, (b) identify the distribution of costs stratified by age and fracture incidence, and (c) identify any cost differences between women receiving drug therapy for osteoporosis management compared to women not receiving drug therapy. Although the exact dollar amounts and percentages may vary among managed care organizations (MCOs), these findings will likely be generalizable to other mixed-model health maintenance organizations (HMOs). The costs described in this study are from the perspective of an MCO, as derived from actual medical and pharmacy claims data.

\section{Methods}

\section{Patient Selection}

Women between the age of 45 and 65 years as of October 1 , 1997, who were continuously enrolled in a 1.2 million-member mixed-model health plan (located in the Midwest) from October 1, 1997 to September 30, 1999, were identified from medical and pharmacy claims databases. The 2-year extraction period was used to determine continuous eligibility over the extraction and evaluation periods. The records of patients with osteoporosis (International Classification of Diseases-ninth revision [ICD-9] codes 733, 733.01, 733.02, 733.03, 733.09), or osteoporosis-related fractures (ICD-9 codes 733.10, 733.11, $733.12,733.13,733.14,733.15,733.16,733.19)$ were extracted using primary and secondary diagnoses. Corresponding medical claims were extracted by common procedures terminology (CPT) codes.

The study consisted of 2 time periods. The first time period, from October 1, 1997, to September 30, 1998, was the extraction period. Patients were identified and eligible for participation in this study if their medical claims included an ICD-9 code for osteoporosis or osteoporosis-related fracture during this 12 -month period. Patients with both ICD-9 diagnoses of osteoporosis and fracture were placed in the more severe diagnosis group, the fracture group. Only women with an ICD-9 code indicating an osteoporotic-related fracture were identified; other fracture diagnoses related to trauma, accidents, or other non-osteoporosis-related fractures were not part of the inclusion criteria and were therefore excluded from the study. Once patients were identified, medical and pharmacy claims were extracted for the 12-month period from October 1, 1998, through September 30, 1999, to determine cost and utilization outcomes. Continuous eligibility was assumed if a patient had at least one pharmacy claim during each calendar quarter of the 2-year study.

\section{Data Collection}

All corresponding pharmacy and medical administrative claims were collected for members identified for inclusion in the study. For the pharmacy claims, the following information was collected: ingredient cost paid, copayment amount, date filled, days supply, metric decimal quantity, member identification number, new or refill code, patient date of birth and sex, national drug code (NDC), therapeutic class, prescription number, pharmacy identification number, amount paid by health plan, prescriber identification number, and prescriber last name. For the medical claims, the following information was obtained: primary or secondary diagnosis of osteoporosis or osteoporosisrelated fracture, member identification number, place and type of service, allowed amount, date of service, admission and discharge date, provider specialty and type, procedure, specialty, and type codes, and patient date of birth and sex.

\section{Cost Calculations}

Once all the data were collected, a relational database was constructed in Microsoft Access using the member identification numbers of the patients as grouped by ICD-9 codes (osteoporosis-only versus fracture), and the medical and pharmacy claims were integrated into a single database. Actual costs incurred by the MCO were included in the analysis. To accurately capture costs, the allowed amount for medical claims and ingredient cost paid for pharmacy claims were used. The allowed amount is defined as the amount submitted for payment as the predetermined allowable charge agreed upon by the managed health care payer for the service or procedure rendered. The drug cost paid amount is defined as the amount allowed by the MCO for payment before any patient liability (copayment or member cost share) has been applied and does not include the pharmacy dispensing fee or rebates or other postservice discounts.

The CPT codes were reviewed, determined to be either related or unrelated to osteoporosis as indicated by the corresponding ICD-9 codes, and assigned to resource unit groups (outpatient services, inpatient services, laboratory, radiology, ambulance, emergency room visits, home health, pharmacy, and other). Overall costs to manage osteoporosis or osteoporosisrelated fractures were determined, and a mean was used to calculate the cost of treatment pppy. A mean was calculated for drug costs and medical treatment costs, and the associated costs were further categorized into the above-mentioned resource unit groups. The mean costs were then stratified into 4 age groups (45-49, 50-54, 55-59, and 60-64) and fracture incidence. The percentage of patients with osteoporosis or osteoporosis-related fractures receiving drug therapy was also determined. Treatment for osteoporosis was defined as drug therapy that included a pharmacy claim for one or more of the following medications: estrogen, alendronate, raloxifene, and calcitonin. Estrogen products were further broken down to include the following categories: oral estradiol, transdermal estradiol, 


\begin{tabular}{|c|c|c|c|}
\hline & \multicolumn{3}{|c|}{ Primary or Secondary Diagnosis* } \\
\hline & $\begin{array}{l}\text { Osteoporosis } \\
\quad(n=516)\end{array}$ & $\begin{array}{c}\text { Fracture } \\
(n=84)\end{array}$ & $\begin{array}{c}\text { Combined } \\
(\mathrm{N}=600)\end{array}$ \\
\hline Age, year $($ mean $+S D)$ & $57+5$ & $55+6$ & $56+5$ \\
\hline \multicolumn{4}{|l|}{ Age category (number., \%) } \\
\hline $45-49$ & $64(12)$ & $17(20)$ & $81(14)$ \\
\hline $50-54$ & $114(22)$ & $20(24)$ & $134(22)$ \\
\hline $55-59$ & $160(31)$ & $28(33)$ & $188(31)$ \\
\hline $60-64$ & $178(35)$ & $19(23)$ & $197(33)$ \\
\hline Receiving osteoporosis drug therapy & $457(89)$ & $57(68)$ & $514(86)$ \\
\hline
\end{tabular}

conjugated estrogens, conjugated estrogen-progesterone combinations, estropipate, ethinyl estradiol, esterified estrogens, oral estrogen-testosterone combinations. Risedronate claims were not captured in this study because the first approval for risedronate for the treatment of osteoporosis was on April 14, 2000, after the period of the extant study.

\section{Statistical Analysis}

Wilcoxon analysis and chi-square analysis were used to describe the differences in costs between patients with osteoporosis and osteoporosis-related fractures and between patients receiving osteoporotic drug therapy and those not receiving any drug therapy. Wilcoxon analysis was also used to determine cost differences by age distribution. All tests were performed using the Statistical Analysis System for Windows (SAS Institute, Cary, $\mathrm{NC}$ ), version 6.12, on an IBM-compatible personal computer in Microsoft Windows 97 environment. The a priori level of statistical significance was set at 0.05 .

\section{Results}

A total of 600 women, 516 with osteoporosis and 84 with fractures, were identified, reflecting prevalence rates of 4.3 per 10,000 female members in the age range 45 to 65 years and 0.84 per 10,000 female members, in the age range 45 to 65 years, respectively. These 600 members consumed $\$ 4.6$ million in total medical resources and $\$ 411,684$ in direct costs related specifically to osteoporosis. By age category, women aged 55 to 59 years experienced the greatest percentage of fractures, accounting for $33 \%$ of the total (Table 1).

\section{Costs by Indication}

Total direct health care costs. Costs unrelated to osteoporosis (unrelated claims) plus the osteoporosis costs comprise the total direct health care costs to manage members with osteoporosis or osteoporosis-related fractures (Table 2). The total direct-cost categories are broken down into the various service components, including inpatient, outpatient, emergency room and ambulance services, radiology, laboratory, pharmacy, and other. The group receiving drug treatment for osteoporosis demonstrated lower inpatient costs than the group not receiving treatment for osteoporosis ( $\$ 1,475$ versus $\$ 3,872, P=.02$ ). A difference was also demonstrated between drug treatment and notreatment groups for radiology services and pharmacy costs. There was no apparent difference in total direct health care costs between the drug treatment group and no treatment for osteoporosis group (Table 2).

For the fracture group compared to the osteoporosis group, pharmacy costs were significantly higher for the fracture group ( $\$ 1,765$ versus $\$ 1,609$ PPPY; $P=.02$ ). There was no difference in total direct health care costs between the osteoporosis group and the fracture group, and pharmacy costs were the only category of component total direct health care costs in which there was a statistically significant difference between the 2 groups.

Disease-specific costs. For costs related to osteoporotic, disease-specific claims, the group of women receiving treatment for osteoporosis was compared to the group of women not taking any prescription drugs for osteoporosis, and the fracture group was compared to the osteoporosis group. Overall, the average pharmacy costs were lower for the fracture group ( $\$ 176$ PPPY) compared to the osteoporosis group (\$314 PPPY, $P=.0001)$; however, the outpatient and radiology service costs were greater in the fracture group (Table 3 ). In addition, the group not receiving any treatment for osteoporosis exhibited higher inpatient, emergency room and ambulance, and laboratory service costs than the group taking osteoporosis medication. The group not receiving drug treatment incurred higher inpatient costs ( $\$ 296$ versus $\$ 23, P=.0092$ ) and total average disease-specific costs ( $\$ 724$ versus $\$ 679, P=.0001$ ) compared to the group that received drug treatment for osteoporosis (Table 3 ).

Principal cost drivers. Outpatient costs made up the high- 
TABLE 2 Total Direct Health Care Costs* Per Patient Per Year

\begin{tabular}{|c|c|c|c|c|c|c|}
\hline & Treatment & No treatment & $P$ value & Fracture & Osteo-only & $P$ value \\
\hline Service Type & $\mathrm{n}=514$ & $\mathrm{n}=86$ & & $\mathrm{n}=84$ & $\mathrm{n}=516$ & \\
\hline Inpatient Mean (+ SD) & $1475(+6,122)$ & $3,872(+11,563)$ & 0.02 & $2,719(+9,785)$ & $1,672(+6,680)$ & 0.82 \\
\hline Outpatient Mean (+ SD) & $2,692(+4,284)$ & $4,194(+7,061)$ & 0.12 & $3,749(+5,546)$ & $2,770(+4,664)$ & 0.83 \\
\hline ER/ambulance Mean (+ SD) & $36(+111)$ & $37(+118)$ & 0.83 & $51(+123)$ & $33(+110)$ & 0.06 \\
\hline Radiology Mean (+ SD) & $495(+1,262)$ & $958(+3,556)$ & 0.02 & $852(+3,585)$ & $514(+1,272)$ & 0.13 \\
\hline Laboratory Mean (+ SD) & $666(+1,043)$ & $1,187(+2,943)$ & 0.65 & $1,042(+1,964)$ & $692(+1,384)$ & 0.70 \\
\hline Pharmacy Mean (+ SD) & $1,674(+1,593)$ & $1,373(+1,665)$ & 0.0006 & $1,765(+1,844)$ & $1,609(+1,595)$ & 0.04 \\
\hline Other (DME) Mean (+ SD) & $31(+237)$ & $8(+29)$ & 0.13 & $13(+85)$ & $30(+234)$ & 0.93 \\
\hline Total Mean (+ SD) & $7070(+11,477)$ & $11,628(+21,800)$ & 0.65 & $10,191(+18,926)$ & $7,322(+12,399)$ & 0.35 \\
\hline
\end{tabular}

\section{TABLE 3 Disease-specific Direct Health Care Costs* Per Patient Per Year}

\begin{tabular}{|c|c|c|c|c|c|c|}
\hline & Treatment & No treatment & $P$ value & Fracture & Osteo-only & $P$ value \\
\hline Service Type & $\mathrm{n}=514$ & $\mathrm{n}=86$ & & $\mathrm{n}=84$ & $n=516$ & \\
\hline Inpatient Mean (+ SD) & $23(+284)$ & $296(+1,560)$ & 0.0092 & $265(+1,544)$ & $29(+318)$ & 0.09 \\
\hline Outpatient Mean (+ SD) & $139(+315)$ & $161(+295)$ & 0.98 & $225(+456)$ & $129(+281)$ & 0.006 \\
\hline ER/Ambulance Mean (+ SD) & $0.61(+7.2)$ & $2.4(+11.8)$ & 0.01 & $1(+8)$ & $0.83(+8)$ & 0.48 \\
\hline Radiology Mean (+ SD) & $53(+150)$ & $109(+704)$ & 0.001 & $103(+710)$ & $55(+152)$ & 0.0001 \\
\hline Laboratory Mean (+ SD) & $119(+200)$ & $154(+371)$ & 0.087 & $168(+390)$ & $117(+195)$ & 0.98 \\
\hline Pharmacy Mean (+ SD) & $343(+264)$ & NA & 0.0001 & $176(+205)$ & $314(+277)$ & 0.0001 \\
\hline Other (DME) Mean (+ SD) & $0.76(+10.1)$ & NA & - & $\mathrm{NA}$ & $0.76(+10)$ & - \\
\hline Total Mean (+ SD) & $679(+706)$ & $724(+2,493)$ & 0.0001 & $939(+2,523)$ & $645(+697)$ & 0.05 \\
\hline
\end{tabular}

est percentage of the mean total annual direct medical costs of care for both the fracture and osteoporosis groups, individually and collectively as a whole. By service type for the total healthcare-related costs, outpatient services represented 38\% of the total, inpatient services accounted for $24 \%$, and outpatient pharmacy was $21 \%$. Disease-specific costs associated with osteoporosis were distributed as pharmacy, representing $43 \%$ of the costs, followed by outpatient (21\%), laboratory (18\%), and inpatient (9\%) (Figure 1).

Cost by age category. Average medical costs for women with a fracture were highest for the 60 to 64 years age category ( $\$ 17,403$ PPPY, $P=.0379$ ) (Table 4). The total pharmacy-related costs were highest for women age 55 to 59 years with a fracture $(\$ 2,589$ PPPY, $P=.0137)$. The disease-specific costs broken down by age category showed that women with a diagnosis of osteoporosis in the 45 to 49 years and 50 to 54 years age categories had the highest average pharmacy-related costs when compared to women with a fracture diagnosis ( $\$ 243$ and $\$ 304$ PPPY, respectively; $P<0.05)$. Disease-specific costs for women aged 60 to 64 years with fractures also appeared to be the highest in comparison to the other age categories ( $\$ 1,991 \mathrm{PPPY})$, but these apparent differences were not statistically significant (Table 4).

Cost by drug use. A total of $86 \%(n=514)$ of women were receiving osteoporosis medication, while $14 \%(n=86)$ were not; $32 \%$ of women with a history of fractures were not receiving drug therapy, while $11 \%$ of women with an osteoporosis diagnosis were not on drug therapy. Women in the 60 to 64 years age category not receiving osteoporosis drug therapy incurred the highest average cost PPPY $(\$ 18,592)$ compared to women not receiving drug therapy $(\$ 5,074, P=.0156)$. Overall, women not receiving osteoporosis treatment appeared to have slightly higher average total medical costs ( $\$ 11,628$ versus $\$ 7,070)$, but this apparent difference was not statistically significant (Table 2). Women receiving drug treatment for osteoporosis had lower total disease-specific direct health care costs compared to women who did not received drug treatment for osteoporosis ( $\$ 724$ versus $\$ 679, P=.0001$ ) (Table 4 ). 


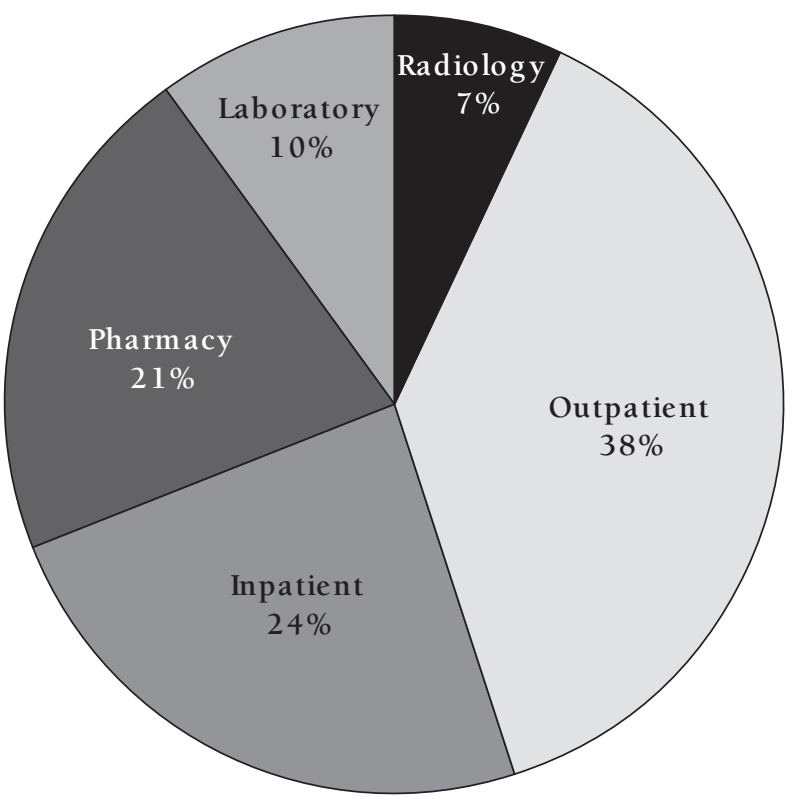

Percentage of Total Health Care Costs

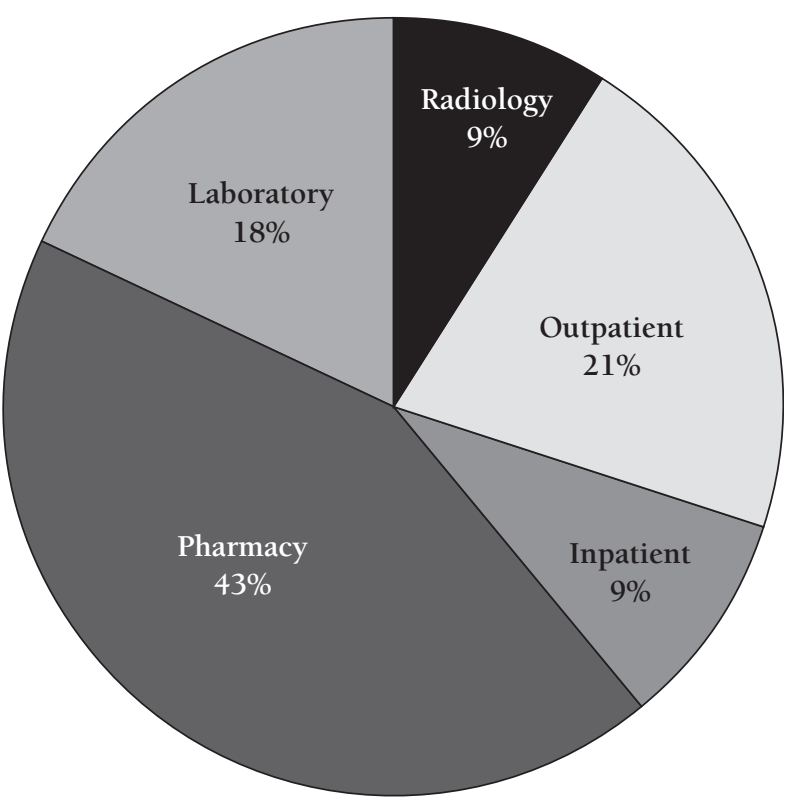

Percentage of Disease-Specific Health Care Costs
Breakdown of osteoporosis drug treatment. Forty-one percent of the patients taking drug therapy for osteoporosis were on estrogen or estrogen combination (i.e., progestin or testosterone combination) alone, followed by alendronate monotherapy $(17 \%)$, raloxifene monotherapy (4\%), and calcitonin monotherapy $(6 \%)$. Other treatments included combinations of the above 4 drugs and accounted for the remaining $32 \%$ of the prescription utilization. Estrogen and alendronate taken in combination comprised $18 \%$, and estrogen and calcitonin taken in combination accounted for $5 \%$ of the prescription utilization.

\section{Discussion}

This study identified 600 women with osteoporosis or fracture diagnosis who utilized a total of $\$ 411,684$ in osteoporosis-related health care resources. Approximately $86 \%(n=514)$ of women with a diagnosis of osteoporosis or fracture received drug therapy. Torgerson and Dolan found that among fracture patients (hip, wrist, and spine), only those with a diagnosed vertebral fracture had a significantly higher use of drug therapy (39\% versus 2\%). Furthermore, they found that as many as 59\% of fracture patients were not prescribed antiosteoporotic treatment in the year following the fracture. Although the percentage cited in this study is higher than that reported for the general population, it is not known how many women continue to take therapy as directed over a long period of time. Prior research conducted by Ettinger et al. found that as many as $50 \%$ to $60 \%$ of women initiating hormone therapy discontinue within 3 years. ${ }^{6,7}$

Another reason for the higher percentage of drug therapy use among this population of women in the extant study is due to the inclusion criteria that included at least one pharmacy claim per calendar quarter. Using this criterion to determine eligibility may have selectively excluded members who chose not to obtain drug therapy during the 1 -year observation period. All members with medical coverage were also covered by pharmacy benefits through the same health plan, and it is therefore unlikely that these women were receiving prescriptions from another source not captured by the plans prescription claims.

A more thorough method for determining continuous eligibility would have involved examination of the enrollment database to determine active enrollment. However, this eligibility information was not readily available for analysis in this study.

The mean age of the women in this population was 56.3 years. As expected, older women appeared more likely to incur a fracture attributable to osteoporosis. Women in the 55 to 59 years category experienced the greatest percentage of fractures, accounting for $33 \%$ of the total. A limitation of this study was that women aged 65 years and older were not included as part of this analysis and would have offered an interesting look at fracture incidence and costs in a linear relationship to age. Women age 65 and older were excluded from analysis because access to the complete Medicare claims data was not available and reasonable coordination of benefits was not possible, reducing the certainty of capturing all relevant medical claim records for women age 65 older.

On the basis of mean total costs, women taking osteoporosis medications exhibited lower health care resource consump- 
TABLE 4 Average Medical and Pharmacy-related Costs Per Patient Per Year by Age Category

\begin{tabular}{|c|c|c|c|c|}
\hline \multirow{2}{*}{$\begin{array}{l}\text { Patient Group } \\
\text { Osteoporosis }\end{array}$} & \multicolumn{2}{|c|}{$\begin{array}{l}\text { Average Total Health Care Costs } \\
(\text { Mean + SD) }\end{array}$} & \multicolumn{2}{|c|}{$\begin{array}{l}\text { Disease-Specific Health Care Costs* } \\
(\text { Mean + SD) }\end{array}$} \\
\hline & Medical & Pharmacy & Medical & Pharmacy \\
\hline $45-49(n=64)$ & $6,632+12,657$ & $2,020+2,190$ & $332+650$ & $243+231 \dagger$ \\
\hline $50-54(\mathrm{n}=114)$ & $6,506+13,688$ & $1,773+1,650$ & $382+690$ & $304+257 \dagger$ \\
\hline $55-59(\mathrm{n}=160)$ & $5,435+11,973$ & $1,396+1,150 \dagger$ & $261+362$ & $299+268$ \\
\hline $60-64(n=178)$ & $5,125+10,005 \dagger$ & $1,546+1,539$ & $362+769$ & $358+306$ \\
\hline \multicolumn{5}{|l|}{ Fracture } \\
\hline $45-49(n=17)$ & $3,921+4,702$ & $1,239+1,375$ & $291+427$ & $84+165 \dagger$ \\
\hline $50-54(n=20)$ & $5,802+9,433$ & $1,172+971$ & $230+189$ & $118+100 \dagger$ \\
\hline $55-59(n=28)$ & $6,942+11,080$ & $2,589+2,538 \dagger$ & $596+903$ & $240+250$ \\
\hline $60-64(n=19)$ & $17,403+33,886 \dagger$ & $1,648+1,268$ & $1,991+5,057$ & $227+213$ \\
\hline
\end{tabular}

* Disease specific refers to costs specifically related to osteoporosis or fracture-related costs.

+ Statistical significance $(\mathrm{P}<.05)$ when women in age categories compared between osteoporosis and fracture groups.

Pharmacy costs were compared to pharmacy costs and medical costs were compared to medical costs.

tion in inpatient, emergency room and ambulance, and radiology services than those women not taking any drug treatment for osteopososis. Women with fractures were less likely to be receiving osteoporosis-related drug treatment $(68 \%$ versus $89 \%$ ) than their osteoporosis counterparts without diagnoses for fractures. Interestingly, women with fractures incurred lower disease-specific pharmacy costs but had higher general pharmacy costs than women with osteoporosis. The need to manage other disease states that were a consequence of the fracture, such as pain, antithrombotic therapy, and depression, is the obvious explanation for this finding. However, further analysis is warranted to determine the differences in the types of prescription utilization accounting for the overall higher pharmacy costs in women with fracture compared to osteoporosis-only.

When comparing total health-care-related costs between the 2 groups of women, osteoporosis and fracture, pharmacy costs were significantly higher for the fracture group, but only $68 \%$ of the patients in the fracture groups were receiving drug therapy to prevent osteoporosis-related fracture. The major cost drivers for both groups included outpatient services (38\%), followed by inpatient services (24\%) and pharmacy (21\%). When diseasespecific costs were calculated for both groups, the major cost drivers were found to be pharmacy costs (43\%), followed by outpatient $(21 \%)$, laboratory (18\%), and inpatient services (9\%). The differences in the identified cost drivers when accounting for only osteoporosis-specific health care utilization may be attributed to the criteria used to identify osteoporosisrelated costs. Pharmacy costs were derived from prescriptions for drugs used to treat osteoporosis. With regard to coding practices, not all physician offices code diagnoses in the same manner on medical claims. This practice may result in variations in coding. Finally, members were identified by primary and sec- ondary diagnosis codes. Members with multiple disease states with more than 2 diagnosis codes may not have been captured in the dataset due to the method of patient identification used in this study. The results of our study may have been more representative and reliable if we had expanded our search method to include all diagnoses submitted on the medical claims. This method may have captured more osteoporosis-specific claims. Given these factors, the cost estimates for disease-specific osteoporosis or fracture events may have been underestimated.

Estrogen therapy was the most prevalent drug therapy among women in this study population, followed by alendronate, calcitonin, and raloxifene. In this study, women receiving osteoporosis drug treatment, on average, had lower medical-related health care costs than their non-drug-therapy counterparts. The 60 to 64 years age group not receiving drug therapy had greater than 3 times the average total cost PPPY $(\$ 18,592)$ than women taking osteoporosis drug therapy $(\$ 5,074, P=.0156)$. This result may suggest that the use of prescription drugs is associated with lower medical and other health-care-related costs. For osteoporosis-specific medical costs, no statistically significant differences were found between users and nonusers of osteoporosis drug therapy. However, average medical costs were lower for the group utilizing osteoporosis-specific drug therapy. Further investigation is needed to conclusively show that use of an osteoporosis drug may be positively correlated with a decrease in health care costs and resource utilization.

While estrogen replacement therapy (ERT) and opposed estrogen replacement therapy, otherwise know as hormone replacement therapy (HRT), have long been considered to be the most effective for maintenance of bone density and the development of stronger bones, recent studies have suggested 
that the risk of harm from prolonged HRT may outweigh its benefits. ${ }^{1,89}$ Combined estrogen/progestin therapy (HRT) has been associated with unwarranted adverse effects such as vaginal bleeding, breast tenderness, increased risk of cardiovascular disease, and breast cancer. ${ }^{8}$ Recent findings from the Women's Health Initiative (WHI) study assessed the effects of combined HRT in healthy postmenopausal women with an intact uterus. ${ }^{8,9}$ These findings will most likely decrease the use of postmenopausal hormones for the prevention of osteoporosis. The WHI study was designed to assess major health benefits and risks of the most commonly used combined hormone preparation in the United States. The study showed no benefit for the prevention of coronary heart disease and indicated a small, but significant, increase in the risk of cardiovascular events for women taking the combined HRT. Women on the combined HRT had a 22\% increased risk of cardiovascular disease, including a 29\% increased risk of coronary heart disease.

The WHI study was also designed to determine the incidence of breast cancer as a primary adverse outcome of combined therapy. The WHI study found a 26\% increase in the risk of breast cancer, confirming previously observed findings. It is important to note that while there was a statistically significant increased risk of cardiovascular disease and breast cancer in WHI, the absolute increased risk for individual women was small and estimated to be 7 more coronary heart disease events (37 versus 30) per 10,000 women per year and 8 additional new cases (38 versus 30 ) per 10,000 women per year, respectively.

Of equal or perhaps greater importance, the WHI study showed positive correlation between use of HRT and the decreased risk of vertebral and other osteoporotic fractures. The rates of hip fracture decreased by $34 \%$, confirming that HRT has beneficial impact on bone mineral density. The WHI study was the first clinical trial to date to demonstrate the protective effect of HRT in the prevention of fractures secondary to osteoporosis.

While the data from the WHI study are suggestive and the findings statistically significant, the results from the study pertain only to women taking combined continuous conjugated equine estrogen $(0.625 \mathrm{mg} /$ day $)$ and medroxyprogesterone acetate ( $2.5 \mathrm{mg} /$ day), and the conclusions can be applied only to this formulation. Based on the findings from the WHI study, users of HRT seeking protective benefits for osteoporosis prevention may turn to alternative therapies, including the bisphosphonates or selective estrogen receptor modulators. ${ }^{8}$ If women also have vasomotor menopausal symptoms, HRT or ERT are of obvious benefit but should be evaluated for risk versus benefit for each individual patient. ${ }^{9}$

Many health plans and employer groups are seeking to develop WHI programs intended to increase awareness of issues surrounding women's health among members and physicians. Possible interventions include identification of candidates who would benefit from bone-strengthening drug therapy. Health plan sponsors may also want to identify women currently on HRT to help physicians assess reasons for use and to evaluate potential benefits versus risks and alternative therapies. The decision to use HRT is patient-specific and requires close examination of the risk versus benefit in each individual. Nondrug interventions include fall-avoidance education, exercise programs, and nutritional supplementation, including calcium and vitamin D. Increase in the use of bisphosphonates and selective estrogen receptor modulators may become more apparent to pharmacy benefit managers and have profound financial impact on costs of the pharmacy benefit as these drugs replace some of the ERT and HRT use.

\section{Limitations}

This study was based on a 1-year snapshot of retrospective claims data. Using claims experience to study groups for the purpose of making comparisons can be challenging because the data contained within medical and pharmacy claims do not contain laboratory values and other information about the health status of the study population. There may have been population-based differences in demographics between the various groups of women that we studied (osteoporosis versus fracture diagnosis and treatment versus no treatment) that we were not able to account for because we used medical claims data. We were unable to control for demographic differences between the groups. Also, we chose a 1-year period of time to study patterns of resource utilization and cost differences among the groups of women. This time period is not long enough to draw definitive conclusions, and therefore, needs to be studied further by others. For example, it is possible that women in the osteoporosis-only group had, in fact, incurred an osteoporosisrelated fracture prior to the data capture period if an ICD-9 code for fracture was not identified during the extraction phase. This is one of the limitations of a retrospective claims-based study.

The absence of a control group makes our results suggestive. We are unable to make definitive conclusions regarding the costs of care without a control group. The sample sizes between the groups of women were also not standardized because we did not have control over how many women would be identified by the criteria that we established at the beginning of the study. This is another limitation of using medical and pharmacy claims data. Our results may also be influenced by the characteristics of the patients enrolled in this health plan. Since this health plan serves mostly employer groups, the women enrolled may be younger, healthier, and more active, and women aged 65 years or older were excluded from analysis because we were unable to obtain reliable Medicare medical claims records for them.

Our method of cost analysis attempted to capture direct medical and pharmacy costs to reflect the total direct cost burden, including member cost amounts (e.g., medical visit and prescription copayments). These are discounted direct medical costs obtained through MCO negotiation with hospital, medical, and pharmacy providers.

\section{Conclusion}

The average direct health care costs were highest for women aged 60 to 64 years presenting with fractures compared to women in 
the same age category with osteoporosis only. In order of magnitude, the cost drivers for osteoporosis were outpatient services, inpatient services, and pharmacy costs. Women not receiving drug therapy for osteoporosis treatment incurred slightly higher average total health care costs than women receiving drug therapy. The results in the study are suggestive that treatment with osteoporotic agents may offset use of disease-specific health care resources and costs. Further research in this area is warranted to make a definitive conclusion. Unhealthy patients incur a greater direct cost burden to health plans. The use of preventive health care may offset the long-term consequences of disease and improve health status. One of the primary concerns for MCOs is the allocation of resources for preventive care to maintain or improve health status and thereby offset the long-term cost of treating diseases with avoidable adverse consequences.

\section{ACKNOWLEDGMENTS}

The authors acknowledge the assistance of Sandra D. Cassard, ScD, and Clare Wheelock, VP, Database Development, AdvancePCS.

\section{DISCLOSURES}

The work of author Shetal S. Desai was funded, in part, by an educational grant from Abbott Laboratories. This article was sponsored by the AdvancePCS Center for Women's Health. The study was independent research conducted as a residency project during Managed Care Residency at AdvancePCS and was presented at the American Society of Health-System Pharmacist's 57th Meeting in Philadelphia, PA, June 6, 2000. Desai served as principal author of the study and was responsible for study concept and design and drafting of the manuscript. Critical revision of the manuscript was the work of Desai and author Babette S. Duncan. Analysis and interpretation of data was primarily the work of Desai and Sandra D. Cassard. Statistical expertise was contributed by Cassard. Author Alice S. Sloan reviewed the study and manuscript and provided health plan information and demographics. Administrative, technical, and/or material support was provided by Clare Wheelock, Jennifer Freas, and AdvancePCS.

\section{REFERENCES}

1. National Osteoporosis Foundation. Physician's Guide to Prevention and Treatment of Osteoporosis. Washington, D.C.: National Osteoporosis Foundation; 1999.

2. Ray NF, Chan JK, Thamer M, et al. Medical expenditures for the treatment of osteoporotic fractures in the United States in 1995: report from the National Osteoporosis Foundation. J Bone Miner Res. 1997;12:24-35.

3. Sanders KM, Nicholson GC, Ugoni AM, et al. Health burden of hip and other fractures in Australia beyond 2000: projections based on the Geelong Osteoporosis Study. MJA. 1999;170:467-70.

4. Sendi P, Palmer AJ. Modeling the socioeconomic impact of osteoporosisrelated hip fractures in Switzerland. Osteoporos Int. 2000;11:92-94.

5. Torgerson DJ, Dolan P. Prescribing by general practitioners after an osteoporotic fracture. Ann Rheum Dis. 1998;57:378-79.

6. Ettinger B, Li D-K, Klein R. Continuation of post-menopausal hormone replacement therapy: comparison of cyclic versus continuous combined schedules. Menopause. 1996;3:185.

7. Hoerger TJ, Downs KE, Lakshmanan MC, et al. Healthcare use among U.S. women aged 45 and older: total costs and costs for selected postmenopausal health risks. J Women's Health. 1999;8(8):1077-89.

8. Risks and benefits of estrogen plus progestin in healthy postmenopausal women: principal results from the Women's Health Initiative randomized controlled trial. JAMA. 2002;288:321-33.

9. Response to Women's Health Initiative study results by the American College of Obstetricians and Gynecologists; August 2002. 